\title{
Providing Different Quality of Service Levels by Routing Protocol for Virtualized Wireless Sensor Networks
}

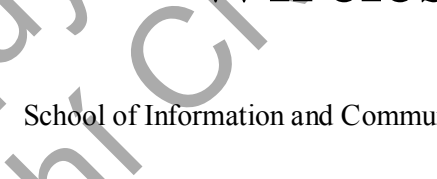
(

(1) (WSN) is a new concept that can provide a common platform upon which new federated sensor architectures can be built, experimented and evaluated. A Virtualized Sensor Network (VSN) is formed by a subset of sensor nodes of a physical WSN that is dedicated to a certain task or an application at a given time. In this paper, we investigate the requirements that virtualization imposes on the routing procedure of the involved WSNs and propose a routing scheme: Virtualized-EMRP (based on EMRP [1]). In addition, over the same WSN, different applications may require different QoS levels but satisfying this is not always a straightforward task. In the context of VSN, this task can be resolved by initializing different instances of Virtualization-EMRP routing protocol and adjusting its routing metrics. We use OMNET++ simulator for different scenarios of Virtualized-EMRP associated with different routing metrics and simulation results show that QoS differentiation for different applications can be achieved.

\section{Keywords - QoS, Virtualization, Wireless Sensor Networks.}

\section{INTRODUCTION}

Nowadays, a WSN operates independently as an isolated island and most of the sensed data is not shared among different administrative domains. The reasons for using dedicated sensor networks include the limited sensing, processing and communication abilities of the nodes, severe power constraints and most of all, the lack of algorithms, protocols and techniques for deploying complex sensor networks. Under a wide variety of conditions (large scale network of thousands nodes, crowded urban area or difficult terrains), independent sensor networks dedicated to a specific task may not be the best or the most practical deployment technique. That is why it is necessary to deploy a WSN that is capable of sharing their physical resources or exchanging information over geographical isolated areas. This concept is called Virtual Sensor Networking [2]. In principle, a Virtual Sensor Network (VSN) consists of sensor nodes providing the ability to be leveraged by a multitude of different administrative domains, platforms, communication protocols and services. In a virtualized sensor networks, new applications can be served without requiring installing new sensors but just reusing existing ones.

For this important purpose of VSN, the most objective of VSN is the resource virtualization. In other words, network and node resources play a very significant role for virtualization of WSN. Given that the VSN system will consist of heterogeneous devices with different capabilities and communication resources, resource virtualization mission are:

Communication resources/connectivity: As the heterogeneous devices of the system may also differ in their communication capabilities, to optimize the overall network performance, routing can take advantage of the heterogeneous neighbor capabilities. Thus, the sensor transmission range or the supported wireless interface will be considered as an attribute taken into account when routing decisions are made.

Dynamic resource control: The (heterogeneous) devices included in a VSN may be battery or mains powered. As the energy consumption depends on a number of operational parameters, these may be tuned dynamically to prolong the network lifetime.

Hardware node resources: affect the complexity of the routing protocol that can be executed. For example, given that security is one of the key requirements, the implementation of trust logic can be 
distributed among nodes in a proportional to hardware resources manner.

Security services virtualization: Different security levels have to be supported to improve the probability of reaching the destination for special purpose messages. These messages may include alarm related messages, service discovery messages which are of higher priority (more vital) in some applications than regular messages carrying sensed data.

Energy level/node status: To improve the network reliability, it is required that the nodes are able to report their energy status and energy level. This information is also valuable for the routing protocol, since the knowledge on neighbor's energy levels can help to significantly save energy consumption. These information are exchanged in VSN nodes for the routing layer to prolong the network life time.

Scalability and mobility: To benefit from the virtualization of the wireless sensor nodes and network, all the nodes involved in the VSN have to cooperate for trust building and routing purposes. Although virtualization targets the reduction of the number of devices installed in an area by exploiting already installed sensors, in this uncontrolled environment, the number of nodes in the neighborhood can fluctuate. Thus, it is mandatory for the relevant protocols to support both scalability and mobility.

From this point of view, virtualization of WSN aims at exploiting the dynamic symbiotic relationships of applications and virtualization requirements for producing a carefully crafted platform of sensor nodes [3] [4]. In other words, a complex and heterogeneous large scale VSN imposes requirement of energy efficiency, seamless connectivity, operational reliability and security [5] [6]. In order to satisfy these requirements, virtualization process will influence the design support of layer 3 (routing) of the protocol stack significantly. In addition, the routing decision of a virtualized routing protocol can influence the application's achieved quality of service (QoS) directly. In many cases, we realize that over a same WSN infrastructure, different applications require different QoS levels. Satisfying this is not always a straightforward task. In order to overcome this problem, we can think of choosing appropriate routing metrics of virtualized routing protocol for handling traffic of these applications differently.

Routing design plays an important role for VSN. However, to the best of our knowledge, the impact of virtualization on routing is rarely investigated recently. In this paper, we investigate the requirements that virtualization imposes on the routing protocol EMRP [1] and extend it to the new Virtualized-EMRP version for supporting different applications in VSNs. We also examine how different QoS levels for applications can be offered by adjusting its routing metrics. Our approach is validated using computer simulations.

The rest of the paper is organized as follows: in Section II, the sensor network virtualization concept is discussed to draw its implications on routing. In Section III we describe the functionalities that need to be carried out in the different nodes of the network by Virtualized-EMRP protocol. Section IV provides simulation results to show how different adopted metrics can lead to different performance aspect optimization over the same sensor network. Finally, conclusions are drawn in Section V.

\section{ROUTING IN VSN}

In this section, the requirements that virtualization impacts the design of a routing protocol are investigated.

\section{A. Requirements for routing design}

First, requirements include the resource virtualization targeting a more efficient use of the available VSN resources and supporting different applications over a same physical WSN. Depending on application's requirements, a virtualized routing scheme must determine which type of resource it needs to be virtualized. To achieve such a goal, each node will be associated with a set of node's attributes describing its resources (hardware, connectivity, energy, and possibly security/trust related functionality). Based on the type of resource, it is possible to select appropriate node's attributes (also called routing metrics) for this routing scheme. Secondly, a VSN routing protocol has to define how 
the information regarding, this virtualized resources will be exchanged, what are the functionalities that need to be carried out in different nodes of the network. Finally, the algorithms that will use this information to turn it into benefits need to be designed carefully in order to achieve the requirements of applications. In order to satisfy these requirements of virtualization, VSN introduces the notion of routing instances and object functions.

\section{B. Object function design}

An important feature that is crucial for supporting virtual wireless sensor networks is that it supports the construction of multiple routing trees with the same or different root nodes based on different routing metrics, which form the so-called routing instances. Routing instance plays an important role in VSN because it helps to build different paths from sensor nodes to root for different applications simultaneously over the same physical WSN. In addition, for achieving different application's performance levels at each time, it is very important to select a set of parameters (routing metrics) that will affect routing decision for each routing instance in the network, called object function. An object function is a function of routing metrics and depending on the requirements of virtualized applications, object functions must be designed carefully. By using object functions of different routing metrics for routing instances, it is possible to provide resource virtualization and define how the information regarding this virtualized resources will be exchanged. Based on selecting appropriate object functions for routing instances, different QoS performance levels for different applications can be achieved.

It is stated that some link or node characteristics may be used as routing metrics, while others can be used as constraints, depending on the design approach. In particular, this is applicable to the dynamic metrics which must be carefully applied (and updated), since they might lead to routing instabilities. Below, the list of routing parameters that can be used for the objective function are presented [7]:
Hop Count (HC): The hop count value is based on reporting the number of traversed nodes along the path to the BS. This is an integer value that can be used as a metric by a node in order to make the forwarding decision. Hop count is an important value since it can help towards reducing transmissions from node to Base Station (BS) and thus contribute towards extending network lifetime and reducing delay. Hop Count, in some cases, can be also represented by Distance.

Expected Transmissions (ETX): This value indicates the expected number of transmissions required for a packet to reach the BS. In some cases, ETX is another value that can be used to quantify the link reliability between two neighboring nodes and thus can be used as an input for reliable routing decision purposes. In this case, ETX is a cross layer metric, since it requires information from the PHY layer component.

Packets Forwarding Indication (PFI): The Packets Forwarding Indication is a measure based on the packets transmitted from a node to its parent and the packets forwarded by the parent node along the route. Despite ETX, PFI can be proved very useful in the presence of malicious nodes.

Residual Energy (RE): Taking into consideration that energy expenditure is of critical importance in WSN deployments, energy (both residual energy and power mode) can be seen as a mandatory metric. Nodes should be able to select a different route to accomplish their tasks, taking into account the residual energy of their neighbors. The frequency of this change must take into account two contradicting issues: route stability and network lifetime.

\section{VIRTUALIZED-EMRP}

In this paper, we are interested in geographical overlapped applications, in which two or more geographical overlapped networks of heterogeneous sensors need to be deployed. There are certain benefits from the collaboration of the networks by virtualization, mainly in the reduction of the number of sensors of each type without losing accuracy or degrading the required user functionality. 


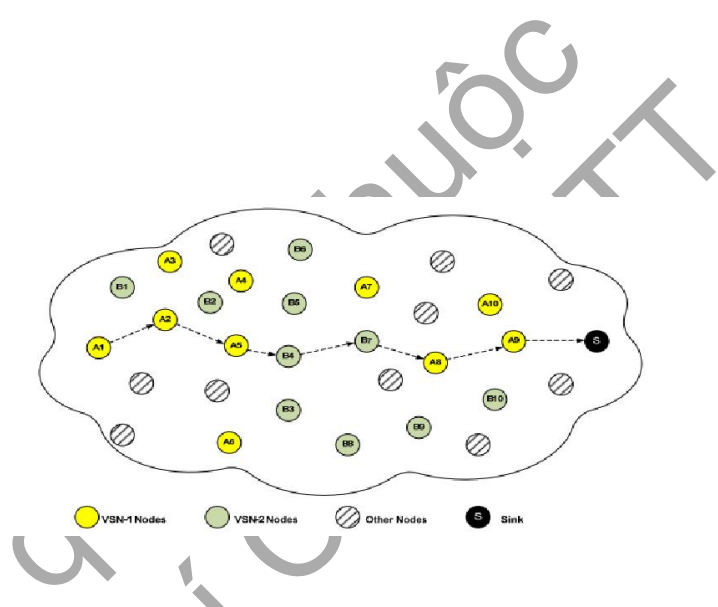

Fgure 1. Geographical VSN

In this section, we describe an use case of geographical application with two VSNs for monitoring the sliding of rock $\mathrm{VSN}_{1}$ and movement of animal $\mathrm{VSN}_{2}$ where virtualization requires an efficient network resource sharing. Furthermore, the area of $\mathrm{VSN}_{1}$ that covers rock sliding can be more dangerous than area of $\mathrm{VSN}_{2}$. Hence, it is easier to remove or change the nodes of $\mathrm{VSN}_{2}$ than $\mathrm{VSN}_{1}$. In order to achieve this purpose, it is necessary to provide better resource utilization (or network lifetime) for $\mathrm{VSN}_{1}$ than $\mathrm{VSN}_{2}$. Finally, the sliding of rock is more important than the animal movement so that and $\mathrm{VSN}_{1}$ needs to have better rate delivery ratio than $V S N_{2}$. These three features (efficient resource sharing, better network lifetime and better rate delivery for $\mathrm{VSN}_{1}$ than $\mathrm{VSN}_{2}$ ) are the most important tasks of this use case.

In order to achieve efficient network resource sharing, a virtualized routing scheme needs to satisfy the following feature: when an event occurs in a VSN and its energy level is negligible, nodes need to be able to relay data over nodes with more energy of other VSN. To achieve such a goal, obviously, a relay node has to be selected based on energy level or energy level is considered the most important parameter when selecting path towards BS. In other words, routing protocol implemented should be energy aware. From this point of view and as described in previous sections, we realize that EMRP (described in [1]) satisfies these characteristics because in EMRP, paths towards BS consist of relay nodes $(\mathrm{RN})$ and backup nodes $(\mathrm{BN})$ that are selected based on a function of energy. That means the nodes with more energy are chosen for data transmission towards BS. This relay node selection process of
EMRP helps to relay data over nodes with more energy in other VSN for achieving network resource sharing [8].That is why we select and extend EMRP for virtualization purpose, called Virtualized-EMRP routing protocol. In order to provide better resource utilization (or network lifetime) and better rate delivery ratio for $\mathrm{VSN}_{1}$ than $\mathrm{VSN}_{2}$, we propose the initialization of different instances per application and the use of different routing metrics per instance to achieve the desired QoS differentiation. VirtualizedEMRP routing protocol that satisfies the above described criteria is described in the next section.

\section{Virtualized-EMRP protocol:}

For geographical applications, different physical VSNs are fixed for one application from beginning.

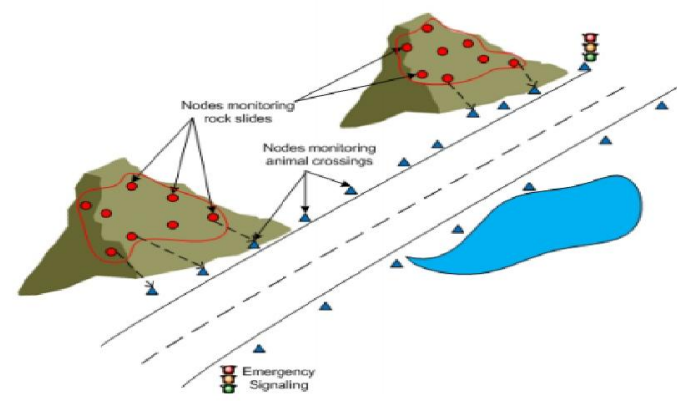

Figure 2. Rock sliding and animal movement

In the application in Figure 2, we have two VSNs $\left(\mathrm{VSN}_{1}\right.$ and $\left.\mathrm{VSN}_{2}\right)$ : each VSN can contain one or many instances. One instance is a set nodes with the same routing policy and utilizes only one object function. Note that in this case, VSNs are fixed but its instance can be setup dynamically in order to achieve different QoS requirement of application (rate delivery ratio, network lifetime...) or instance of this VSN can cover nodes of other VSN. In order to implement EMRP in the context of virtualization, Virtualized-EMRP needs to be able to support different instances per application.

Furthermore, in order to achieve efficient network resource sharing, better resource utilization for $\mathrm{VSN}_{1}$ than $\mathrm{VSN}_{2}$ and better rate delivery ratio for $\mathrm{VSN}_{1}$ than $\mathrm{VSN}_{2}$, Virtualized-EMRP routing protocol has to consider different instances object functions that consists of different routing metrics. In this paper, we do not concentrate on metrics that relate to the quality 
of transmission medium such as ETX and PFI. As described in previous section, energy should be the most important metric for achieving efficient network resource sharing. But using only energy cannot provide different levels of network resources (network lifetime) and rate delivery ratio for $\mathrm{VSN}_{1}$ and $\mathrm{VSN}_{2}$. Hence for this type of application, routing metrics of Virtualized-EMRP routing protocol can be extended to node residual energy and also number of hops or distance. Based on these two metrics, we need to build different object functions for different instances.

It is necessary to recall that in EMRP, there exist two types of object functions: cluster formation function $\mathrm{F}_{\mathrm{CH}}$ and relay node function $\mathrm{F}_{\mathrm{RN}}$. The original version of EMRP utilizes energy, distance and angle value for $\mathrm{F}_{\mathrm{RN}}$. For $\mathrm{F}_{\mathrm{CH}}$, it implements residual energy and intensity of signal sensed. In Virtualized-EMRP, it is necessary to modify these two object functions $\mathrm{F}_{\mathrm{CH}-\mathrm{i}}$ and $\mathrm{F}_{\mathrm{RN}-\mathrm{i}}$ for each instance numbered $i$. The operation of Virtualized-EMRP protocol is described as follows:

\section{a. Initialization phase:}

Unlike the original version of EMRP, in Virtualized-EMRP, it is not necessary to specify Relay Node (RN) and Backup Node (BN). The selection of $\mathrm{RN}$ and $\mathrm{BN}$ is realized by $\mathrm{CH}$ later when $\mathrm{CH}$ needs to transmit data to the BS (in the data transmission phase).In the initialization phase, BS generates BROADCAST_INFO message that contains information relating $\mathrm{VSN}_{1}$ and $\mathrm{VSN}_{2}$ in the networks. Node receives this message will calculate distance from it to the BS. Based on its position, it can specify $\mathrm{VSN}_{1}$ or $\mathrm{VSN}_{2}$ that it belongs to and also instance identification of this VSN. In this application case, InstanceID 1 is set for VSN $\mathrm{VS}_{1}$ and InstanceID 2 is set for $\mathrm{VSN}_{2}$. For each instance, a cluster formation object function and relay node object functions (described in the transmission phase) are defined in order to achieve the better network lifetime and better rate delivery ratio for $\mathrm{VSN}_{1}$ than $\mathrm{VSN}_{2}$.

\section{b. Cluster formation phase:}

When an event occurs, node that senses this event will identify its VSN based on its position and also specify instance identification of this VSN. Obviously, node will know which cluster formation function it should use later.

If an event occurs in $\mathrm{VSN}_{\mathrm{i}}$ with instance identification $i$, it will utilize object function $i$ defined for this instance identification.

$$
\begin{gathered}
F_{C H-i}(j)=f(j) \\
\operatorname{MaxF}_{C H-i}(j) \rightarrow C H
\end{gathered}
$$

where $j$ is the node numbered $j$ and $i$ is instance identification. $\mathrm{CH}$ is the node with maximum cluster head object function. Obviously, this $\mathrm{CH}$ can specify the VSN and instance identification that it belongs to.

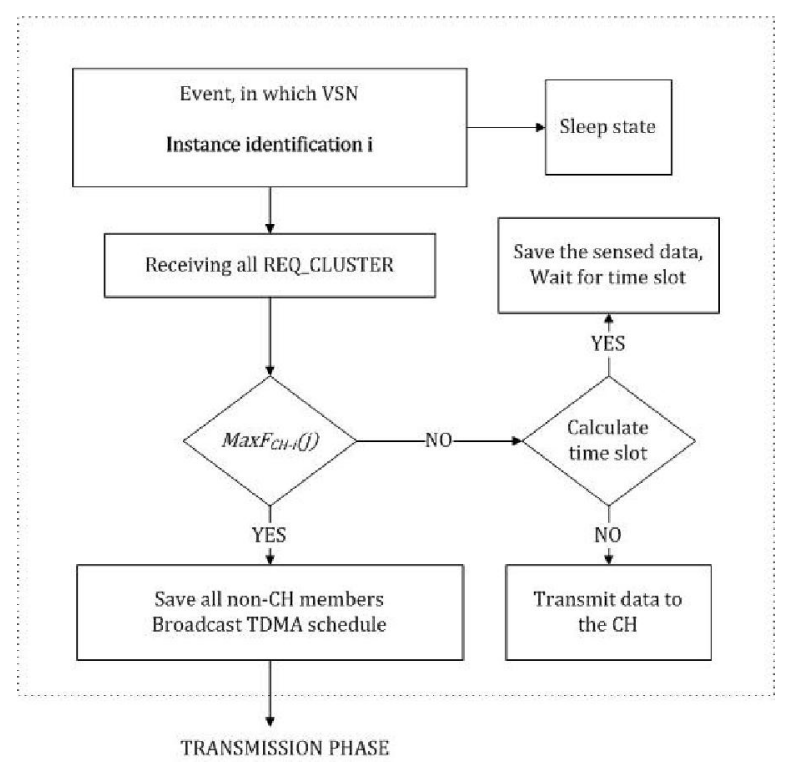

Figure 3. Cluster Formation stage

\section{c. Data transmission phase:}

In EMRP protocol, data transmission belongs to only one application. In Virtualized-EMRP, it can be done simultaneously for different application's instances. That is why the data transmission phase in Virtualized-EMRP needs also to be modified in order to adapt to this change.

After receiving messages from all non- $\mathrm{CH}$ members, $\mathrm{CH}$ needs to select $\mathrm{RN}$ and $\mathrm{BN}$ in order to transmit data to the BS. Based on the previous phase, $\mathrm{CH}$ knows already the VSN it needs to work for, its instance identification and associated object function. $\mathrm{CH}$ then broadcasts REQUEST_RELAY message to 
all other nodes within its range. Each node that receives the REQUEST_RELAY message calculates the value of relay node function based on the object function of instance, puts the result into an ACK_RELAY message, and sends it back to the CH. Then $\mathrm{CH}$ will wait for receiving all ACK_RELAY messages from RN candidates and checks if it can transmit data to the BS directly. Otherwise it performs relay node function and the nodes that have the maximum value and second maximum value of relay node function are selected as $\mathrm{RN}$ and $\mathrm{BN}$.$$
F_{R N-i}(j)=f(j)
$$$$
\operatorname{Max}_{R N-i}(j) \rightarrow R N
$$$$
\operatorname{SecondMax} F_{R N-i}(j) \rightarrow B N
$$

where $j$ is the node numbered $j$ and $i$ is instance identification. $\mathrm{RN}$ and $\mathrm{BN}$ are the nodes with maximum and second maximum relay node object function.

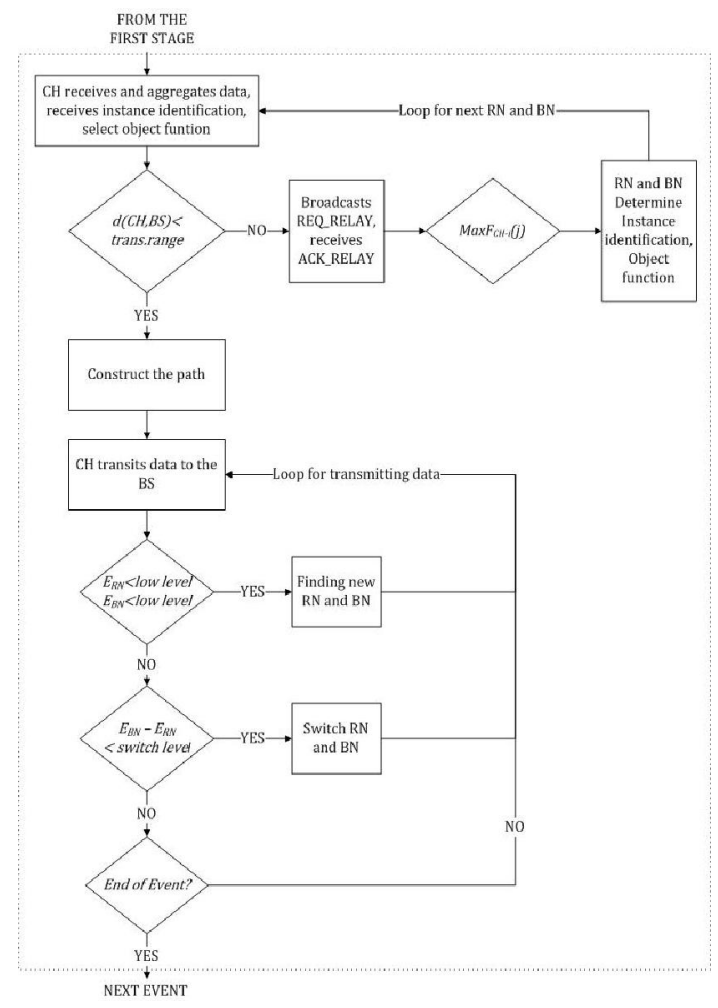

Figure 4. Flowchart of Data Transmission

After selecting $\mathrm{RN}$ and $\mathrm{BN}, \mathrm{CH}$ then transmits DATA_TO_BS message that contains not only aggregated data but also the instance identification of VSN of this event to its $\mathrm{RN}$. The RN receives this information will continue to select next $\mathrm{RN}$ and $\mathrm{BN}$. By doing this, all RNs and BNs on the path towards $\mathrm{BS}$ are informed about the instance identification and object function used for this instance. Next RN and $\mathrm{BN}$ can work with the same object function designed for this instance identification and this process stops only when $\mathrm{RN}$ or $\mathrm{BN}$ reaches the $\mathrm{BS}$.

\section{PERFORMANCE EVALUATION}

The proposed Virtualized-EMRP scheme was implemented in OMNET++ simulator which is a public-source, component-based, modular and openarchitecture simulation environment with strong GUI support and an embeddable simulation kernel. We simulate here two VSNs that are distributed randomly in an area of $(500 * 1000) \mathrm{m}^{2}$ (see the following figure). Each VSN has 100 nodes. The purposes of virtualization here are described in the following:

- Efficient network resource sharing (represented by number of relaying messages)

- Provide better network resource for $\mathrm{VSN}_{1}$ than $\mathrm{VSN}_{2}$ (represented by network lifetime)

- $\quad$ Provide better rate delivery ratio for $\mathrm{VSN}_{1}$ than $\mathrm{VSN}_{2}$.

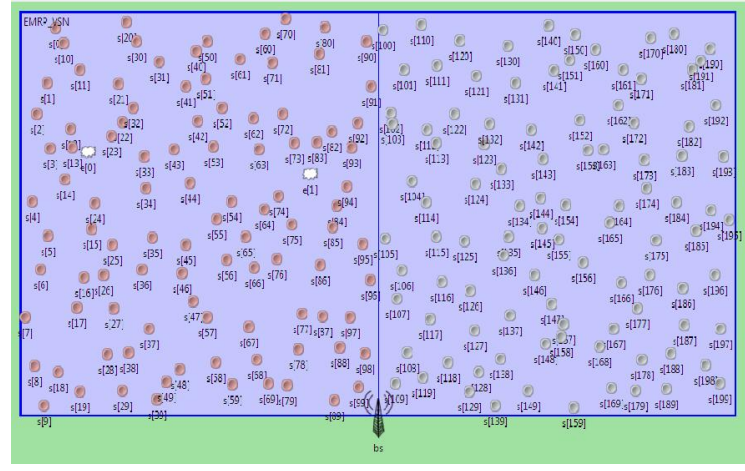

Figure 5. Simulated topology

The parameter setup for simulation is described in the following table. $\mathrm{VSN}_{1}$ consists of pink sensors while $\mathrm{VSN}_{2}$ consists of gray ones. The following table describes parameters used in our simulation: 
Research, Development and Application on Information and Communications Technology

TABLE I. SimULATION PaRaMeters

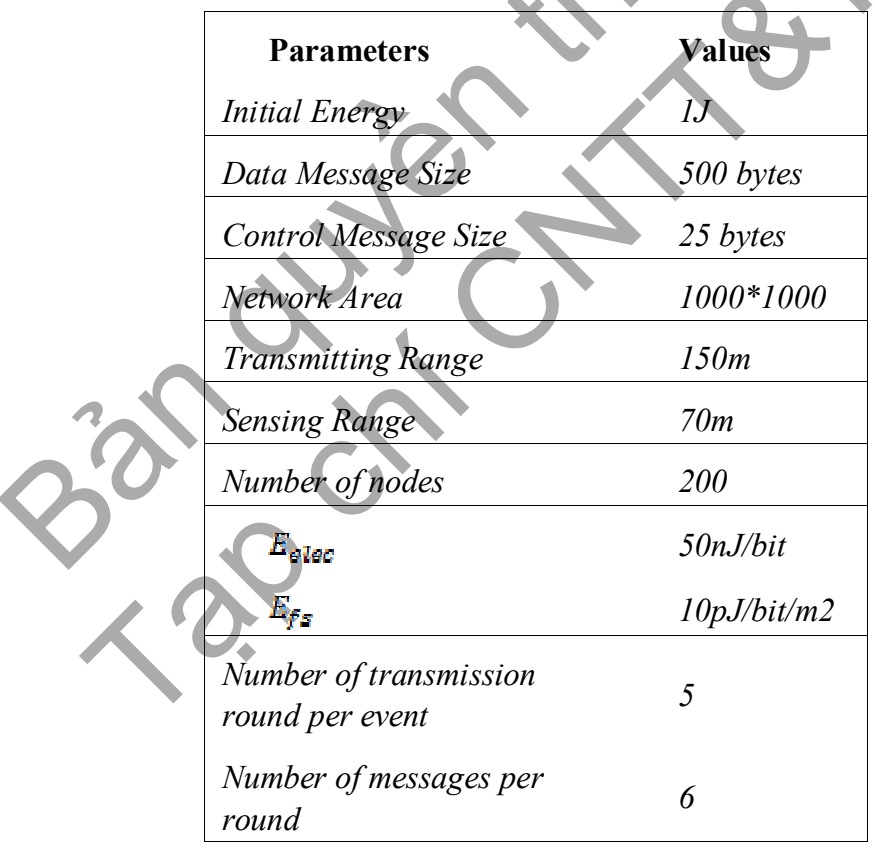

We assume that the switch level is $0.5 \%$ of the initial energy and the low level is $10 \%$ of the initial energy. A node will stop participating in routing process if its energy falls below $1 \%$ of the initial energy. The simulation is run until all the sensor nodes that have distance to BS less than their transmission range die. That means all the links to BS are failed and data transmission process cannot continue. We measure three performance metrics: relaying message number, network lifetime and rate delivery ratio. To calculate the energy dissipation for transmitting and receiving packets, we use the same conditions describing as follows:

- Energy used for transmitting packets comprises of transmitted energy $E_{T x}=k * E_{\text {elec }}$ and energy required to amplify signal $E_{\text {amp }}=k^{*} E_{f s} * d^{2}$ where $E_{\text {elec }}$ is energy of transceiver electron and $E_{f s}$ is energy of transmission in free space model and $d$ is the distance from sending node to receiving nodes.

- $\quad$ Energy used for transmitting packets:

$$
E_{T x}(k, d)=E_{T x}+E_{a m p}
$$

- $\quad$ Energy used for receiving packets:

$$
E_{R x}(k)=k * E_{\text {elec }}
$$

A. Scenario 1: In this scenario, we have $\mathrm{VSN}_{1}$ and $\mathrm{VSN}_{2}$ that are represented by two instances with identifications 1 and 2 respectively. In order to satisfy the QoS requirement described previously, we select two object functions as follows:

For instance 1:

$$
\begin{aligned}
& F_{C H-1}(j)=E(j) /\{d(j, B S) * d(j, \text { Event })\} \\
& F_{R N-1}(j)=E(j) * \cos a(j) / d(j, B S)
\end{aligned}
$$

For instance 2:

$$
\begin{gathered}
F_{C H-2}(j)=1 /\{d(j, B S) * d(j, \text { Event })\} \\
F_{R N-2}(j)=\cos a(j) / d(j, B S)
\end{gathered}
$$

While $E(j)$ is the residual energy of node $j$, $d(j$, Event $)$ is distance from node $j$ to the event, $d(j, B S)$ is distance from node $j$ to $\mathrm{BS}, a(j)$ is angle value between $\mathrm{CH}, j$ and BS.

Nodes in $\mathrm{VSN}_{1}$ work for instance numbered 1 and select only the first function of cluster head and relay node. Nodes belong to $\mathrm{VSN}_{2}$ can work for both instances and select both the first and second functions of cluster head and relay node. We propose to use for instance numbered 1 cluster head selection function and relay node selection function that are based on both distance and residual energy while object function of instance numbered 2 depends only on distance. By doing that, routing decision for instance 1 is better than for instance 2 . We calculate the number of relaying messages, network living time and failure link ratio of two VSNs.

TABLE II. NUMBER OF RELAYING MESSAGES

\begin{tabular}{|l|c|c|}
\hline \multicolumn{1}{|c|}{ Messages Number } & $\mathbf{V S N}_{\mathbf{1}}$ & $\mathbf{V S N}_{\mathbf{2}}$ \\
\hline Total messages number & 7469 & 7125 \\
\hline $\begin{array}{l}\text { Number of relaying messages } \\
\text { from } \mathrm{VSN}_{1} \text { to } \mathrm{VSN}_{2} \text { and from }\end{array}$ & 180 & 0 \\
$\mathrm{VSN}_{2}$ to $\mathrm{VSN}_{1}$ & & \\
\hline
\end{tabular}

In the above table, the numbers of relaying message are presented. Because nodes of $\mathrm{VSN}_{2}$ can work for both instances, $\mathrm{VSN}_{2}$ can transmit messages originated from $\mathrm{VSN}_{1}$ (180 messages) while the total message numbers of both VSNs are 7469 and 7125 respectively. This number of relaying messages 
proves that $\mathrm{VSN}_{2}$ can share its resource for $\mathrm{VSN}_{1}$ when necessary. Later, we concentrate on evaluating network lifetime of both VSNs. Network lifetime is represented by two metrics: First node dead and Total live round. Total live round is the network operation time until all nodes are out of energy and this metric is calculated by number of rounds. First node dead is the number of round when the first node in the network runs out of energy. All these two parameters are plotted in the following figure. From this figure, we can easily realize that total live round of $\mathrm{VSN}_{1}$ is better than $\mathrm{VSN}_{2}$. For the first node dead parameter, it is also similar. That means the quality of service of $\mathrm{VSN}_{1}$ is better than $\mathrm{VSN}_{2}$.

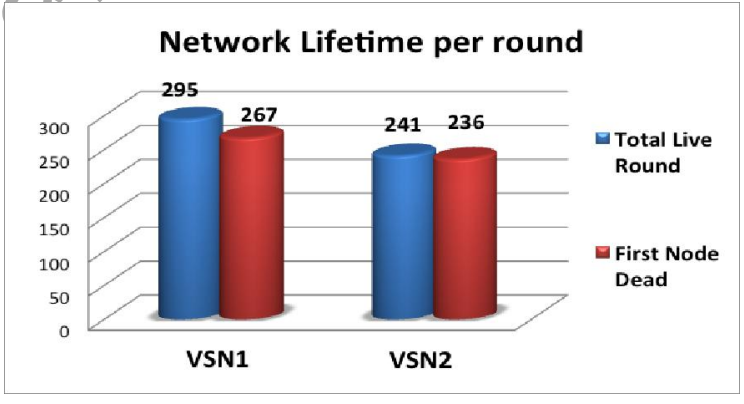

Figure 6. Network Lifetime

Now the rate delivery ratio of this scenario is measured and evaluated. The rate delivery ratio is calculated as the ratio between number of messages that are transmitted successfully to the BS and the total number of messages. The following table presents this performance:

TABLE III. RATE DELIVERY OF SCENARIO 1

\begin{tabular}{|c|c|c|c|}
\hline VSN & $\begin{array}{c}\text { Total } \\
\text { number of } \\
\text { frame }\end{array}$ & $\begin{array}{c}\text { Number } \\
\text { of faillure }\end{array}$ & $\begin{array}{c}\text { Rate } \\
\text { Delivery } \\
\text { Ratio (\%) }\end{array}$ \\
\hline$V S N_{1}$ & 7467 & 3 & 99.96 \\
\hline$V S N_{2}$ & 7125 & 330 & 95.37 \\
\hline
\end{tabular}

As shown in this table, $\mathrm{VSN}_{1}$ achieves better rate delivery ratio than $\mathrm{VSN}_{2}$. More concretely, the rate delivery ratio of $\mathrm{VSN}_{1}$ is $99.6 \%$ and of $\mathrm{VSN}_{2}$ is $95.37 \%$.

For conclusion, $\mathrm{VSN}_{1}$ transmits only data of its event to the BS while $\mathrm{VSN}_{2}$ transmits data of events of both $\mathrm{VSN}_{1}$ and $\mathrm{VSN}_{2}$. That is why energy consumption of $\mathrm{VSN}_{1}$ is better than $\mathrm{VSN}_{2}$ or $\mathrm{VSN}_{1}$ achieves better energy efficiency. In addition, the routing metric of $\mathrm{VSN}_{1}$ depends on energy and distance or the use of residual energy of routing metric of $\mathrm{VSN}_{1}$ leads to better routing decision than $\mathrm{VSN}_{2}$.

\section{B. Scenario 2:}

In this scenario, network composes of two instances numbered 1 and 2. Each node can work paralely for both two instances. Instance numbered 1 supports both routing metrics distance and node residual energy while instance numbered 2 supports only distance.

The first instance implements following cluster head and relay node function:

$$
\begin{gathered}
F_{C H-1}=E(j) /\{d(j, B S) * d(j, \text { Event })\} \\
F_{R N-1}=E_{\max } / K / E(j)+d(j, B S) / d(j, C H)+\operatorname{sina}(j)
\end{gathered}
$$

For instance 2:

$$
\begin{gathered}
F_{C H-2}=1 /\{d(j, B S) * d(j, \text { Event })\} \\
F_{R N-2}=d(j, B S) / d(j, C H)
\end{gathered}
$$

While $E(j)$ is the residual energy of node $j$, $d(j$, Event $)$ is distance from node $j$ to the event, $d(j, B S)$ is distance from node $j$ to $\mathrm{BS}, a(j)$ is angle value between $\mathrm{CH}, j$ and $\mathrm{BS}$.

Compared to the scenario 1 , every node of two VSNs can work for both instances. In this case, the function of cluster formation is similar to the one used in scenario 1 , but the function of relay node is not the multiplication of residual energy, distance and angle value but the sum of them. This can lead to better performance (please see [9] for more details). The relay node function of instance 2 depends only on distance while the relay node function of instance 1 depends also on residual energy and distance. That is why the routing decision made by instance 1 is better than instance 2 and it leads to the better network lifetime and rate delivery ratio of $\mathrm{VSN}_{1}$ than $\mathrm{VSN}_{2}$. The following table presents number of relaying message over other VSN of this scenario. The number of relaying message over other VSN in this case are 481 and 600 (from $\mathrm{VSN}_{1}$ to $\mathrm{VSN}_{2}$ and 
Research, Development and Application on Information and Communications Technology

from $\mathrm{VSN}_{2}$ to $\mathrm{VSN}_{1}$ ). This performance metric proves that both VSNs can share network resources for each other.

TABLE IV. NUMBER OF REL. MESSAGES

\begin{tabular}{|c|c|c|}
\hline \multicolumn{1}{|c|}{ Messages Number } & $\mathbf{V S N}_{\mathbf{1}}$ & $\mathbf{V S N}_{\mathbf{2}}$ \\
\hline Total messages number & 7753 & 7399 \\
\hline $\begin{array}{l}\text { Number of relaying messages } \\
\text { from } V \mathrm{SN}_{1} \text { to } \mathrm{VSN}_{2} \text { and from } \\
\mathrm{VSN}_{2} \text { to } \mathrm{VSN}_{1}\end{array}$ & 474 & 567 \\
\hline
\end{tabular}

Now, we analyze the network lifetime of each VSN, which is presented in Figure 7. From this figure, it is clear that $\mathrm{VSN}_{1}$ achieves better total live round and first node dead metric than $\mathrm{VSN}_{2}$.

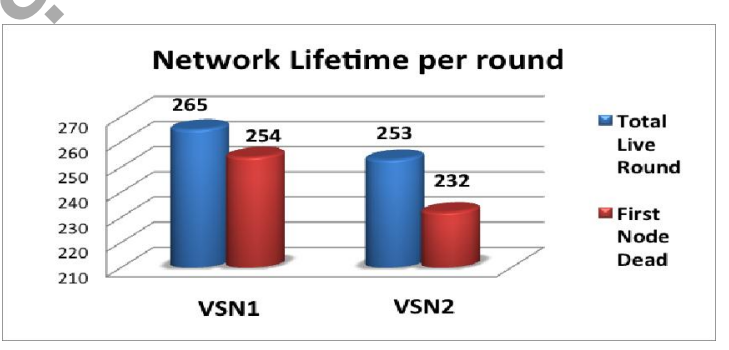

Figure 7. Network lifetime

Next, we evaluate rate delivery of this scenario. As shown in this table, $\mathrm{VSN}_{1}$ achieves better rate delivery ratio than $\mathrm{VSN}_{2}$. This result can be explained simply because the routing metric of $\mathrm{VSN}_{1}$ depends on node residual energy, its routing decision is also better than $\mathrm{VSN}_{2}$. That is why network lifetime and rate delivery ratio of $\mathrm{VSN}_{1}$ is better than $\mathrm{VSN}_{2}$.

TABLE V. RATE DELIVERY OF SCENARIO 2

\begin{tabular}{|c|c|c|c|}
\hline VSN & $\begin{array}{c}\text { Total } \\
\text { number of } \\
\text { mesages }\end{array}$ & $\begin{array}{c}\text { Number } \\
\text { of faillure } \\
\text { messages }\end{array}$ & $\begin{array}{c}\text { Rate } \\
\text { Delivery } \\
\text { Ratio (\%) }\end{array}$ \\
\hline$V S N_{1}$ & 7753 & 52 & $99.33 \%$ \\
\hline$V S N_{2}$ & 7399 & 371 & $94.99 \%$ \\
\hline
\end{tabular}

\section{Comparison:}

In both scenarios, the first scenario achieves better QoS differentiation between two VSNs or better purpose of prolonging network lifetime and rate delivery ratio for $\mathrm{VSN}_{1}$ than second scenario. The reason of this characteristic is that in the second scenario, nodes can transmit data for both events rock sliding and animal movement and work with both object functions. That is why the differentiation in this scenario is not so clear as the first scenario.

\section{CONCLUSION}

In this paper, we investigate the impact of virtualization on routing procedure for VSN. Different issues related to the object functions, virtualized routing scheme are also discussed. In addition, we are interested in virtualized geographical cases and point out that a virtualized routing protocol needs to achieve efficient network resource sharing, differentiated levels of network resource and differentiated levels of rate delivery ratio. Based on this, we propose a Virtualized-EMRP algorithm that can satisfy all these goals. Different scenarios using Virtualized-EMRP are simulated. Result shows that $\mathrm{VSN}_{1}$ can achieve efficient network resource sharing, better network resource and better rate delivery ratio than $\mathrm{VSN}_{2}$.

\section{REFERENCES}

[1] T. Tran Vinh, T. Ngo-Quynh, M. Banh, "Energy Mesh Routing Protocol for Wireless Sensor Networks," in Proc. of Advanced Technologies for Communications (ATC 2012), 260-270, Oct. 2012.

[2] Islam, M.M.; Hassan, M.M.; Lee, G.-W.; Huh, E.-N. A Survey on Virtualization of Wireless Sensor Networks. Sensors, 12, 2175-2207 (2012).

[3] M. M. Islam, M. M. Hassan, and E. N. Huh, "Virtualization in wireless sensor network: challenges and opportunities," in Proceedings of the 13th International Conference on Computer and Information Technology (ICCIT '10), Dhaka, Bangladesh, December 2010.

[4] S. Kabadayi, A. Pridgen, and C. Julien, "Virtual sensors: abstracting data from physical sensors," in Proceedings of the International Symposium on a World of Wireless, Mobile and Multimedia Networks (WoWMoM '06), pp. 587-592, Buffalo-Niagara Falls, NY, USA, June 2006. 
[5] S. Kabadayi, A. Pridgen, and C. Julien, "Virtual sensors: abstracting data from physical sensors," in Proceedings of the International Symposium on a World of Wireless, Mobile and Multimedia Networks (WoWMoM '06), pp. 587-592, Buffalo-Niagara Falls, NY, USA, June 2006.

[6] J. H. Shin and D. Park, "A virtual infrastructure for large-scale wireless sensor networks," Computer Communications, vol. 30, no. 14-15, pp. 2853-2866, 2007.

[7] EIHAL, THALES and HAI, "Design Principles of TrustDaware Routing Protocol supporting Virtualization", A framework of VITRO project, 2012.

[8] Thu Ngo-Quynh, Dien Nguyen-Quang, “Energyaware Routing Protocol for Virtualization of Wireless Sensor Networks", In Proceeding of the ECTI-CON Conference, May 15-17, Krabi. Thailand, 2012
[9] Thu Ngo-Quynh, Hieu Tran Trung, Vinh Tran Quang, "Improving Energy Efficiency for ARPEES Routing Protocol for Wireless Sensor Networks", In Proceeding of the 2012 International Conferences on Advances Technologies for Communications (ATC 2012), October 10-12, Hanoi, Vietnam, 2012.

\section{AUTHOR'S BIOGRAPHY}

Thu Ngo Quynh receives her $\mathrm{Ph}$. Dr at the Technical University Berlin and is currently working at the School of Information and Communication Technology, Hanoi University of Science and Technology. Her interests are: QoS mechanisms, Multipath and Multichannel in Wireless Sensors Network, Internet of Things... 